\section{$\$$ Research Square}

Preprints are preliminary reports that have not undergone peer review.

They should not be considered conclusive, used to inform clinical practice, or referenced by the media as validated information.

\title{
Unique Immunological Profile In Patients With COVID-19
}

\section{Stefania Varchetta}

Fondazione IRCCS Policlinico San Matteo

\section{Dalila Mele}

Fondazione IRCCS Policlinico San Matteo

\section{Barbara Oliviero}

Fondazione IRCCS Policlinico San Matteo

\section{Stefania Mantovani}

Fondazione IRCCS Policlinico San Matteo

\section{Serena Ludovisi}

Fondazione IRCCS Policlinico San Matteo

\section{Antonella Cerino}

Fondazione IRCCS Policlinico San Matteo

\section{Marco Vecchia}

Fondazione IRCCS Policlinico San Matteo

\section{Silvia Roda}

Fondazione IRCCS Policlinico San Matteo

\section{Michele Sachs}

Fondazione IRCCS Policlinico San Matteo

\section{Raffaele Bruno}

Fondazione IRCCS Policlinico San Matteo

Mario U. Mondelli ( $\nabla$ mario.mondelli@unipv.it)

Fondazione IRCCS Policlinico san Matteo

\section{Research Article}

Keywords: SARS-CoV-2, host immunity, altered immune phenotype in patients

Posted Date: April 20th, 2020

DOI: https://doi.org/10.21203/rs.3.rs-23953/v1

License: (c) (1) This work is licensed under a Creative Commons Attribution 4.0 International License. Read Full License 
Version of Record: A version of this preprint was published at Cellular \& Molecular Immunology on October 15th, 2020. See the published version at https://doi.org/10.1038/s41423-020-00557-9. 


\section{Abstract}

The relationship between SARS-CoV-2 and host immunity is unknown. We show here that patients with COVID-19 had an altered immune phenotype, with an expansion of adaptive FceRIg ${ }^{\text {neg }}$ NK cells, and inflammatory $\mathrm{CD} 14^{+} \mathrm{CD} 16^{+}$monocytes. T cells were reduced and overexpressed the Tim-3 exhaustion molecule. Low frequencies of CD8 T cells and NKG2A ${ }^{+} \mathrm{NK}$ cells, and expansion of mature $\mathrm{CD} 57^{+} \mathrm{NK}$ cells were associated with poor prognosis. These findings unveil a unique immunological profile in COVID-19 patients.

\section{Introduction, Results And Discussion}

Severe Acute Respiratory Syndrome Coronavirus-2 (SARS-CoV-2) is responsible for a pandemic thus far responsible for nearly 1 million cases of Coronavirus Disease-19 (COVID-19) with a case/fatality rate of $4.5 \%$ [1]. The infection usually causes mild symptoms, but may be responsible for severe interstitial pneumonia, myocarditis, acute kidney injury, acute respiratory distress syndrome (ARDS), multiorgan failure and death [2]. Laboratory tests indicate that patients with severe progression of COVID-19 show signs of secondary haemophagocytic lymphohistiocytosis (sHLH), a hyperinflammatory syndrome characterised by a potentially fatal cytokine storm with multiorgan failure, which may be triggered by viral infections [3]. Akin to SHLH, COVID-19 is characterized by lymphopenia, and increased serum ferritin, Ddimer, C-reactive protein (CRP), and lactic- dehydrogenase (LDH), which are also considered predictors of poor outcome [4].

Moreover, several serum cytokine concentrations are increased during COVID-19, supporting the hypothesis that virally driven hyperinflammation plays a key pathogenetic role [2].

Despite clear evidence of ongoing overexuberant inflammation, there are no systematic studies addressing phenotypic and functional alterations of innate and adaptive immune cells, that are likely exposed to a variety of stimuli in COVID-19 patients at presentation. The lack of a comprehensive immunological analysis prompted us to assess the phenotypic and functional status of NK cells, $y \delta T$ cells, monocytes and CD 4 and CD8 T cells in patients presenting with clinically moderate to severe interstitial pneumonia emerging in the setting of COVID-19. Patient clinical details and laboratory findings, as well as peripheral blood mononuclear cells (PBMC) flow cytometric analysis are reported in Supplementary Information, Patients and Methods.

The frequency of NK cells was significantly higher in COVID-19 patients compared to healthy controls, being significantly enriched in mature $\left(\mathrm{CD} 56^{\mathrm{dim}} \mathrm{CD} 57^{+}\right)$NK cells (Fig.1a). Interestingly, there was a relative expansion of $\mathrm{CD}_{57}{ }^{+} / \mathrm{FcER}$ Rly ${ }^{\text {neg }}$ adaptive NK cells compared with non-COVID-19 disease controls and healthy controls (Fig.1a) suggesting a SARS-CoV-2-related expansion of this population, whereas the proportion of $\mathrm{CD} 56^{\text {bright }} \mathrm{NK}$ cells was reduced. An increase in $\mathrm{CD} 16^{+} \mathrm{NK}$ cells was also evident compared with healthy controls (Fig 1a). Notably, the frequency of CXCR6-expressing NK cells was low in COVID-19 patients (Fig.1a), most likely since these cells home to the lungs where they concentrate, their ligand 
CXCL16 being produced in large amounts by alveolar macrophages [5]. Additional changes in NK cells included significant reductions in the frequencies of Siglec-7, DNAM-1, NKG2D, NKp30 (Fig.1a), the latter being particularly evident in the adaptive subset (Fig.1c). Importantly, the frequency of PD-1 positive NK cells was significantly higher in the adaptive compared with conventional NK cells in patients with COVID19 (Fig. 1c). No changes were noted in bulk NK cell expression of NKG2C, NKG2A, GITR, TRAIL, CD69, PD1, TIGIT. The trend noted for TIM-3 was not statistically significant (Suppl. Fig.1a). Of note, although no significant changes in degranulation activity or IFNy production were observed using K562 as target cells (Suppl. Fig.1b), there was an increased ability of NK cells to exert antibody-dependent cell-mediated cytotoxicity (ADCC), a function exquisitely performed by adaptive NK cells [6] (Fig. 1d). The proportions of CD56 bright, NKG2A and NKp46 positive NK cells were significantly lower and the proportion of mature $\mathrm{CD} 57^{+}$cells significantly higher in patients who succumbed compared with those who survived (Fig.2a). The relative frequencies of total $\mathrm{CD} 3^{+}, \mathrm{CD} 4^{+}$and $\mathrm{CD} 8^{+} \mathrm{T}$ cells were significantly lower than healthy controls, although no apparent differences were noted with disease controls (Fig.2b). Patients who died showed a significantly lower frequency of CD8 T cells compared with those who survived (Fig.2a). Moreover, both CD4 and CD8 T cells from COVID-19 patients overexpressed Tim-3 compared with healthy controls, suggesting a pan T-cell exhaustion profile (Fig.2C). No differences were found in CD45RO, HLADR, GITR expression or Treg population frequency (Suppl. Fig.2). Importantly, there was a clear relative expansion of CD14/CD16 double positive monocytes, a phenotype associated with an inflammatory profile (Fig. 2d) [7]. There were no statistically significant changes in the frequency of $ү \delta T$ cells (Fig. 2e). Negative correlations were found between laboratory indicators of severe or progressive disease. Thus, NK cells expressing the activating receptors NKp30 and NKp46, as well as CD45RO ${ }^{+}$and Tim- $3^{+}$CD4 T cells, correlated negatively with LDH (Fig. 2f). A negative correlation was also present between NKp46 NK cells and CRP (Fig. 2g).

Information on PBMC phenotype and function are virtually lacking in patients with COVID-19. Here we had the opportunity to evaluate patients admitted to hospital because of moderate to severe COVID-19 interstitial pneumonia and compared them to a small group of SARS-CoV-2 negative pneumonia and healthy We showed that patients with COVID-19 had a relative expansion of mature adaptive NK cells endowed with ADCC function, which was increased in this setting in line with findings in other viral infections, particularly cytomegalovirus [6]. Other phenotypic features were compatible with a dysfunctional NK cell phenotype, namely the reduced frequencies of Siglec-7-, NKG2D- and NKp30expressing cells $[8,9]$. A recent study addressed the kinetics and breadth of immune responses associated with clinical resolution of COVID-19 in a single patient with relatively mild disease [10]. Antibody-secreting cells appeared at the time of viral clearance together with follicular helper T cells and activated CD4 and CD8 T cells. In contrast, in our patients with moderate to severe interstitial pneumonia, some of whom sadly succumbed, Tim-3 positive exhausted CD 4 and CD 8 T cells largely prevailed at presentation and lower frequencies of $C D 8^{+} T$ cells were linked to poor prognosis. A recent study found lower frequencies of CD8 T cells and NK cells with a relative enrichment of NKG2A-expressing cells which returned to normal after clinical recovery, suggesting rescue of impaired T and NK cell function [11]. Interestingly, although no difference in the frequency of NKG2A-expressing NK cells was found between patients and 
controls in the present study, NKG2A $\mathrm{A}^{+} \mathrm{NK}$ cells were lower in patients who did not survive, suggesting that loss of this inhibitory receptor somehow unleashed NK cells in patients with fatal outcome.

Our study provides important novel insights into the pathogenetic mechanisms of COVID-19, characterized by a rapid expansion of phenotypically mature NK cells persisting at high frequency in patient with poor prognosis. The simultaneously reduced frequency of $\mathrm{CD} 4^{+}$and $\mathrm{CD} 8^{+} \mathrm{T}$ cells expressing the Tim-3 exhaustion marker unveils a multifaceted behavior of the two arms of immunity in this clinical setting. The relative enrichment of inflammatory monocytes lends support to the hypothesis that COVID19 resembles in part to the macrophage-activation syndrome which is thought to be closely related to hemophagocytic lymphohistiocytosis (HLH) [12], an uncommon life-threatening disorder of severe hyperinflammation caused by uncontrolled proliferation of activated lymphocytes and macrophages that secrete high levels of inflammatory cytokines. Of note patterns similar to cytokine storm syndromes have been described for COVID-19 and SARS [2].

It is difficult at this early stage to precisely frame COVID-19 within an immunologically coherent clinical entity. Indeed, several peculiarities have emerged that contribute to the uniqueness of its immune profile. Understanding the dynamics and the quality of immune responses to SARS-CoV-2 will provide invaluable translational information to design effective treatments for this potentially deadly disease.

\section{Methods}

Provided as supplemental file.

\section{Declarations}

Author Contributions: SV and DL designed and performed experiments and critically contributed to drafting the manuscript; BO, SM, AC performed experiments and critically read the manuscript; SL, MV, SR, MS, RB recruited patients, prepared the database and critically read the manuscript, MUM designed and discussed the experiments and wrote the manuscript.

\section{Competing Interests: none.}

Ethics: The study protocol conformed to the ethical guidelines of the 1975 Declaration of Helsinki and was approved by the Institutional Review Board and Ethical Committee of Fondazione IRCCS Policlinico San Matteo (Protocol number 20200033215). All patients provided written or, in case they were unable to sign, verbally witnessed informed consent as per the above study protocol.

\section{References}

1. https://worldometers.info/coronavirus/ 
2. Huang, et al. Clinical Features of Patients Infected With 2019 Novel Coronavirus in Wuhan, China. Lancet 395, 497-506 (2020).

3. Ramos-Casals, et al. Adult Haemophagocytic Syndrome. Lancet 383, 1503-16 (2014).

4. Ruan, et al. Clinical Predictors of Mortality Due to COVID-19 Based on an Analysis of Data of 150 Patients From Wuhan, China. Intensive Care Med. https://doi.org/10.1007/s00134-020-05991-x (2020).

5. Morgan, J. et al. Expression of CXCR6 and its ligand CXCL16 in the lung in health and disease. Clin. Exp. Allergy 35, 1572-1580 (2005)

6. Schlums, H. et al. Cytomegalovirus Infection Drives Adaptive Epigenetic Diversification of NK Cells with Altered Signaling and Effector Immunity 42, 443-456 (2015).

7. Ziegler-Heitbrock, The CD14+ CD16+ Blood Monocytes: Their Role in Infection and Inflammation. J. Leukoc. Biol. 81, 584-92.

8. Varchetta, et al. Lack of Siglec-7 Expression Identifies a Dysfunctional Natural Killer Cell Subset Associated With Liver Inflammation and Fibrosis in Chronic HCV Infection. Gut 65, 1998-2006 (2016).

9. Vitale, et al. An Historical Overview: The Discovery of How NK Cells Can Kill Enemies, Recruit Defense Troops, and More. Front. Immunol. 10, 1415 (2019).

10. Thevarajan, et al. Breadth of concomitant immune responses prior to patient recovery: a case report of non-severe COVID-19. Nat. Med. https://doi.org/10.1038/s41591-020-0819-2 (2020).

11. Zheng, M. et al. Functional exhaustion of antiviral lymphocytes in COVID-19 patients. Mol. Immunol. https://doi.org/10.1038/s41423-020-0402-2 (2020).

12. Fisman, N. Hemophagocytic syndromes and infection. Emerging Infect. Dis. 6, 601-8 (2000).

\section{Covid19 Irccs San Matteo Pavia Task Force}

\section{ID Staff}

Raffaele Bruno, Mario U Mondelli, Enrico Brunetti, Angela Di Matteo, Elena Seminari, Laura Maiocchi, Valentina Zuccaro, Layla Pagnucco, Bianca Mariani, Serena Ludovisi, Raffaella Lissandrin, Aldo Parisi, Paolo Sacchi, Savino FA Patruno, Giuseppe Michelone, Roberto Gulminetti, Domenico Zanaboni, Stefano Novati, Renato Maserati, Paolo Orsolini, Marco Vecchia.

\section{ID Residents}

Marco Sciarra, Erika Asperges, Marta Colaneri, Alessandro Di Filippo, Margherita Sambo, Simona Biscarini, Matteo Lupi, Silvia Roda, Teresa Chiara Pieri, Ilaria Gallazzi, Michele Sachs, Pietro Valsecchi.

\section{Emergency Care Unit}


ECU Staff: Stefano Perlini, Claudia Alfano, Marco Bonzano, Federica Briganti, Giuseppe Crescenzi, Anna Giulia Falchi, Roberta Guarnone, Barbara Guglielmana, Elena Maggi, Ilaria Martino, Pietro Pettenazza, Serena Pioli di Marco, Federica Quaglia, Anna Sabena, Francesco Salinaro, Francesco Speciale, Ilaria Zunino.

ECU Residents: Marzia De Lorenzo, Gianmarco Secco, Lorenzo Dimitry, Giovanni Cappa, Igor Maisak, Benedetta Chiodi, Massimiliano Sciarrini, Bruno Barcella, Flavia Resta, Luca Moroni, Giulia Vezzoni, Lorenzo Scattaglia, Elisa Boscolo, Caterina Zattera, Tassi Michele Fidel, Capozza Vincenzo, Damiano Vignaroli, Marco Bazzini.

\section{Intensive Care Unit}

Giorgio lotti, Francesco Mojoli, Mirko Belliato, Luciano Perotti, Silvia Mongodi, Guido Tavazzi

\section{Paediatric Unit}

Gianluigi Marseglia, Amelia Licari, Ilaria Brambilla

\section{Virology Staff}

Barbarini Daniela, Bruno Antonella, Cambieri Patrizia, Campanini Giulia, Comolli Giuditta, Corbella Marta, Daturi Rossana, Furione Milena, Mariani Bianca, Maserati Roberta, Monzillo Enza, Paolucci Stefania, Parea Maurizio, Percivalle Elena, Piralla Antonio, Rovida Francesca, Sarasini Antonella, Zavattoni Maurizio.

\section{Virology Technical staff}

Adzasehoun Guy, Bellotti Laura, Cabano Ermanna, Casali Giuliana, Dossena Luca, Frisco Gabriella, Garbagnoli Gabriella, Girello Alessia, Landini Viviana, Lucchelli Claudia, Maliardi Valentina, Pezzaia Simona, Premoli Marta.

\section{Virology Residents}

Bonetti Alice, Caneva Giacomo, Cassaniti Irene, Corcione Alfonso, Di Martino Raffella, Di Napoli Annapia, Ferrari Alessandro, Ferrari Guglielmo, Fiorina Loretta, Giardina Federica, Mercato Alessandra, Novazzi Federica, Ratano Giacomo, Rossi Beatrice, Sciabica Irene Maria, Tallarita Monica, Vecchio Nepita Edoardo.

\section{Research Laboratories, Division of Infectious Diseases and Immunology}

Antonella Cerino, Stefania Varchetta, Barbara Oliviero, Stefania Mantovani, Dalila Mele.

\section{Pharmacy Unit}

Monica Calvi, Michela Tizzoni 


\section{Hospital Management}

Carlo Nicora, Antonio Triarico, Vincenzo Petronella, Carlo Marena, Alba Muzzi, Paolo Lago

\section{Data Unit}

Francesco Comandatore, Gherard Batisti Bissignandi, Stefano Gaiarsa, Marco Rettani, Claudio Bandi

\section{Figures}



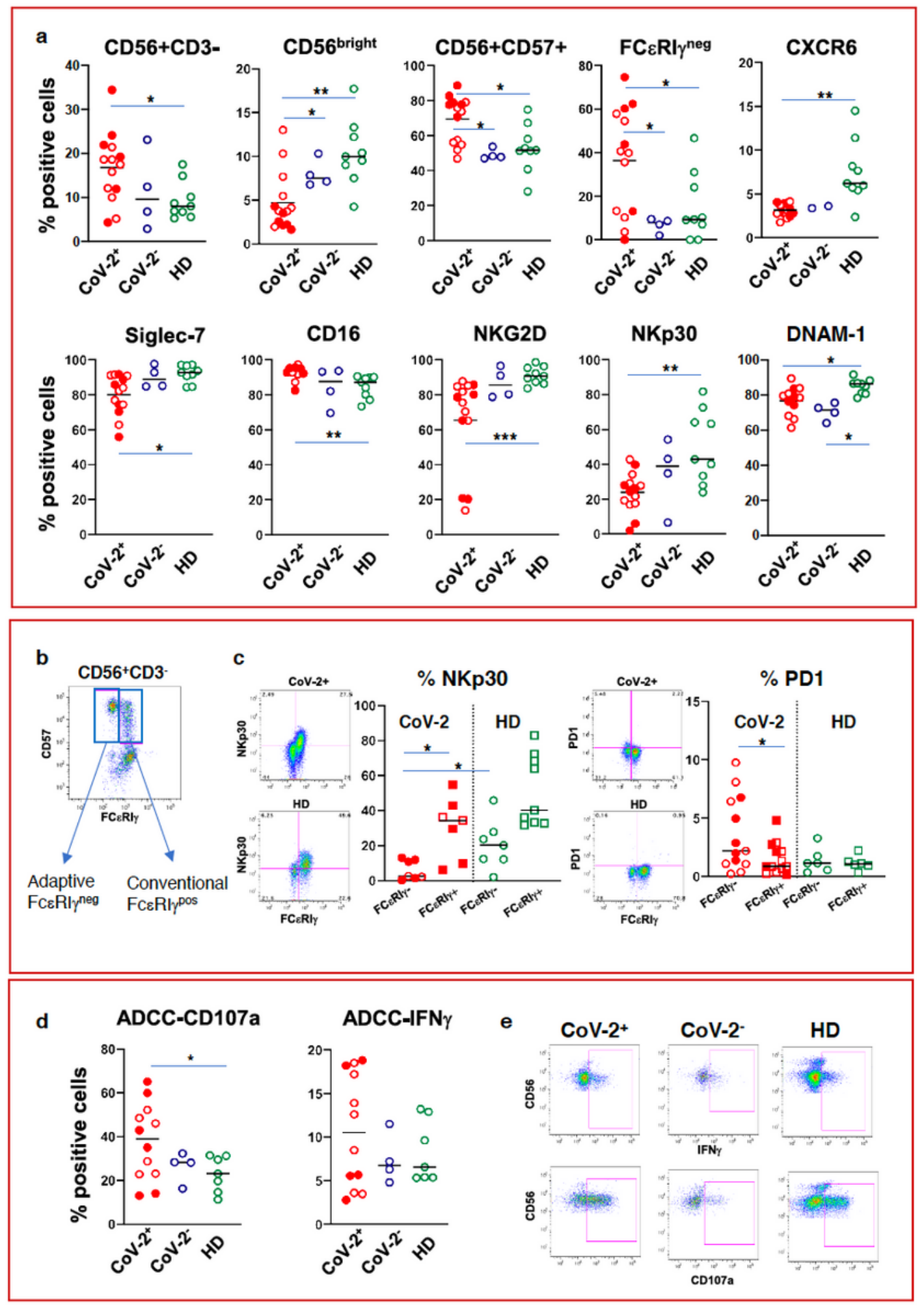

\section{Figure 1}

NK cell characterization in SARS-CoV-2 infection. a) Frequency of NK cells and expansion of mature CD57+ and adaptive (FcERlyneg) NK cells in COVID-19 patients. Reduced frequency of CXCR6, Siglec-7, NKG2D and NKp30, and increased proportion of CD16+ cells. b) Dot plot showing gating on CD57+FcERlyneg adaptive and CD57+FceRlypos conventional NK cells. c) Representative dot plots and graphs showing NKp30 reduction and PD1 increase in adaptive compared with conventional NK cells in 
COVID-19 patients. Representative dot plots are gated on total CD57+ NK cells. Circles indicate adaptive NK; squares, conventional NK. d) Increased NK degranulation and IFNy expression in COVID- 19 patients. e) Representative IFNy and CD107a dot plots in patients and controls. Full red symbols indicate patients who subsequently died. Middle bars represent medians. The One Way Anova test was used to compare three groups. ${ }^{*} p<0.05,{ }^{*} p<0.01, * * * p<0.001$.
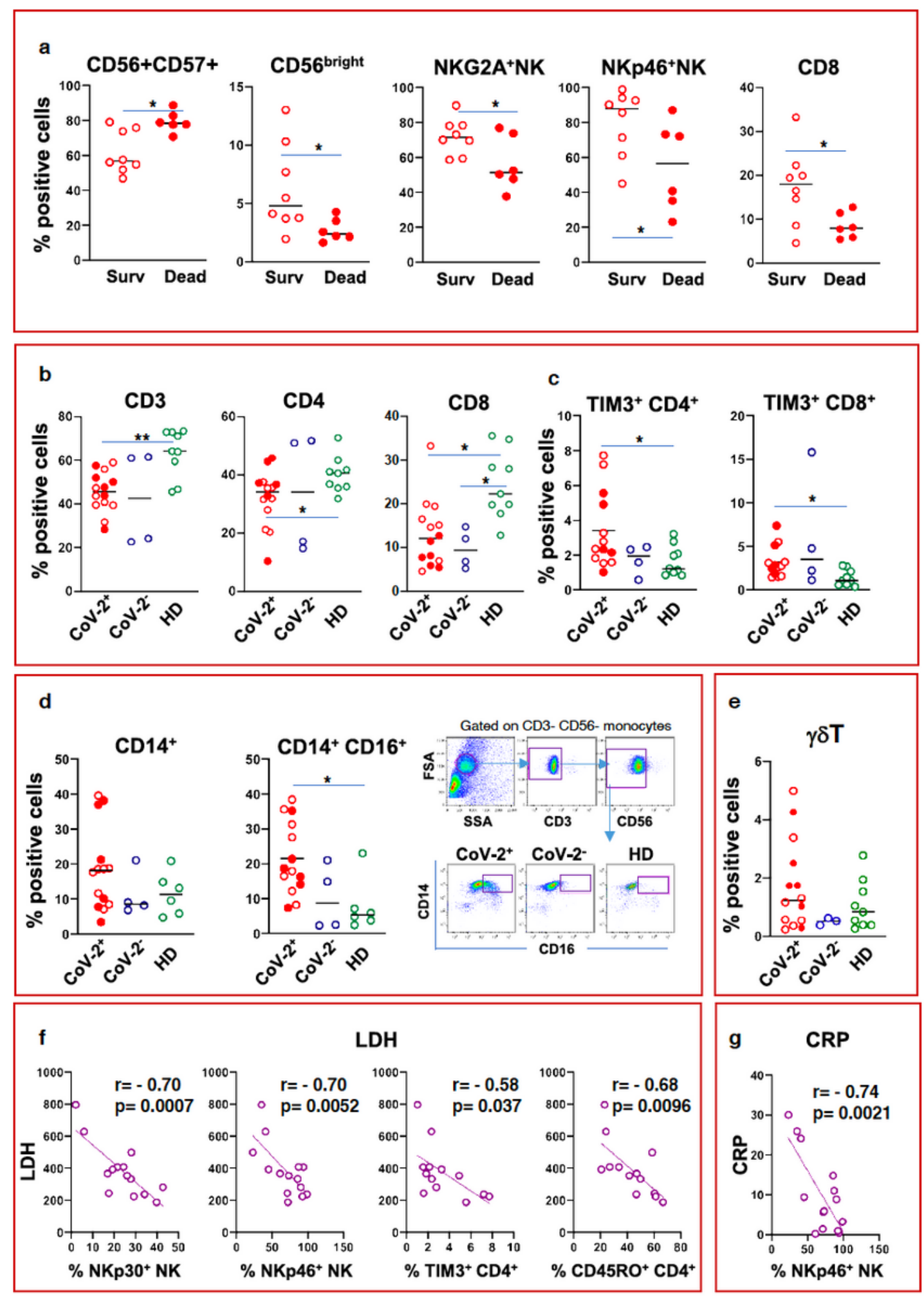

Figure 2 
a) Expansion of mature CD57+ NK cells and reduction of CD56bright NK cells, NKG2A+ and NKp46+ NK cells and CD8 $T$ cells in patients who survived and in those who succumbed. b) Frequencies of total CD3+, CD4+ and CD8+ T cells were reduced in COVID-19 patients compared to HD. c) Tim-3 expressing CD4 and CD8 T cells were increased in COVID-19 patients. d) Expansion of CD14+CD16+ double positive monocytes in COVID-19 patients and representative dot plots. e) No differences were observed in the proportion of $\gamma \delta T$ cells. $f \& g$ ) Correlations of NK and CD4 T receptor molecules with LDH and CRP. Middle bars represent median values. The Mann-Whitney test was used to compare survivors versus dead patients. The One Way Anova test was used to compare three groups. The Pearson test was used to examine correlations. ${ }^{*} p<0.05,{ }^{\star \star} p<0.01$.

\section{Supplementary Files}

This is a list of supplementary files associated with this preprint. Click to download.

- supinfo.pdf 